\title{
Top-down and bottom-up informatics: who has the high ground?
}

Smith, Vincent S. \& Dave Roberts

Natural History Museum, Cromwell Road, London, UK

Recent years have seen the emergence of several major initiatives supporting the development of biodiversity informatics infrastructures. In Europe examples include: $E D I T$ - the European Distributed Institute of Taxonomy; LifeWatch - a biodiversity analytical platform integrating observatories, databases, processing and computational capacity; and SYNTHESYS - funding researcher access to a network of taxonomic collections, laboratories and libraries. These European projects alone represent a spend of approximately 35 Million Euros, with funding contingent on the collaboration of numerous geographically dispersed institutional partners. In this presentation we will review the major informatics products of these initiatives; the different development models used in their construction; and levels of user engagement that provide an indication of their relative success.

A key outcome of these initiatives is the bottom-up emergence of Virtual Research Environments (VRE's) such as the Scratchpad project (http://scratchpads.eu), the EDIT Cyberplatform (http://wp5.e-taxonomy.eu/), and CATE (http://www.cate-project.org/). These tools enable real-time collaboration and dissemination of biodiversity information while simultaneously affording new opportunities for data stewardship, curation, and data mining. Although highly experimental, these environments offer an alternative vision for the practice of biodiversity research that challenges the primacy of traditional publishing as the end point in scholarly communication. Inspired by discourses in the 'Free/Open Source Software' and 'Creative Commons' movements, these tools support the notion of 'Open Science' with its advocacy of more open scientific knowledge production and publishing processes. These VRE's have the potential to be used as instruments of scientific research, blending social and technical solutions to address the challenges that are otherwise insurmountable through individual efforts. The maturity of these tools is dependent upon greater reliability, especially in the data services on which they depend, and through reduced duplication of developer effort. This will deliver an increasing body of users that can have confidence and trust in the systems they use, and who will benefit from the network effects of an enlarged user community. Delivering this requires greater top-down cooperation and integration of our national funding agencies, supporting bottom-up multinational collaborative efforts amongst our research communities. 